\title{
Fpga-Based High Bandwidth Integral Electron Density Interferometer For Mast-U
}

\author{
Kai Jakob Brunner* \\ Durham University \\ E-mail: k.j.brunner@durham.ac.uk \\ Thomas O'Gorman \\ Culham Centre for Fusion Energy \\ E-mail: thomas.ogorman@ccfe.ac.uk
}

\section{Rory Scannell}

Culham Centre for Fusion Energy

E-mail: rory.scannelleccfe.ac.uk

\section{Graham Naylor \\ Culham Centre for Fusion Energy \\ E-mail: graham.nayloreccfe.ac.uk}

\section{Geoffrey Cunningham}

Culham Centre for Fusion Energy

E-mail: Geoffrey.Cunninghameccfe.ac.uk

\section{Ray M. Sharples}

Durham University

E-mail: r.m.sharplesedurham.ac.uk

\section{Nigel A. Dipper}

Durham University

E-mail: n.a.dipper@durham.ac.uk

\begin{abstract}
A multi-MHz bandwidth two-color interferometer to measure the integral electron density has been developed for the Mega Amp Spherical Tokamak - Upgrade (MAST-U). This paper describes the digitization and real time processing part of the system, which is based on field programmable gate arrays (FPGAs) using open source hardware from CERN. The phase shift is measured using heterodyne IQ-downconversion and CORDIC phase rotation enabling detection of integral electron density perturbations above $4 \mathrm{MHz}$. The design is shown to be stable towards $2 \pi$-wraps beyond any rate of density change or mirror movement expected in a fusion device. Data is digitized, processed and streamed out in real time with less than $4 \mu$ s delay making the diagnostic capable of being integrated into real-time Tokamak protection systems and continuousshot experiments.
\end{abstract}

First EPs Conference on Plasma Diagnostics - 1st ECPD,

14-17 April 2015

Villa Mondragone, Frascati (Rome) Italy

* Speaker. 


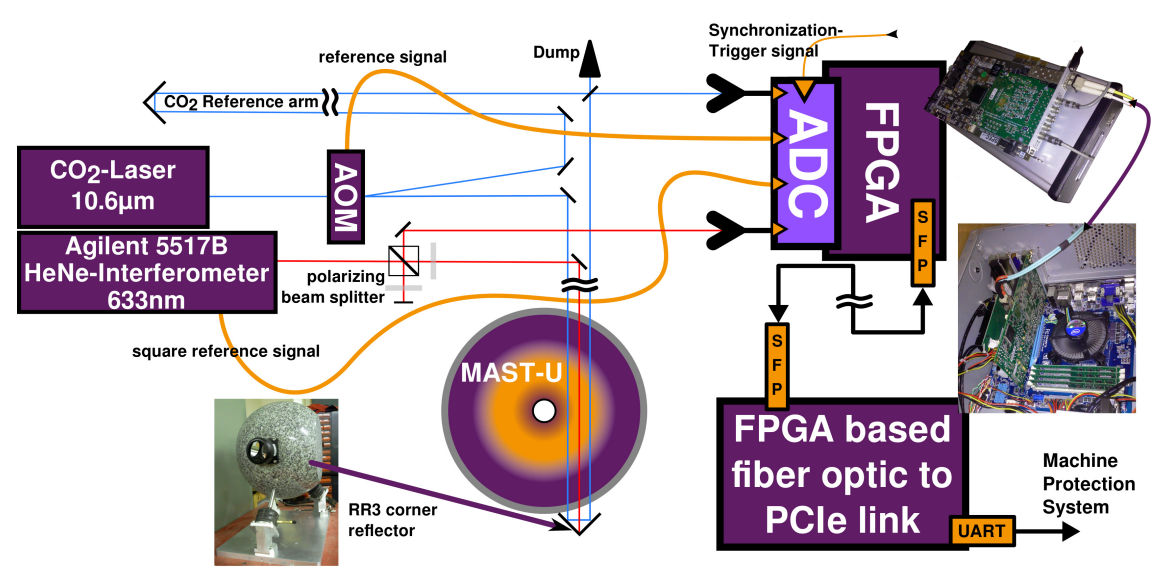

Figure 1: Top level schematic of the diagnostic hardware.

\section{Introduction}

The integral electron density (IED) is one of the fundamental plasma parameters in a fusion device. It is used to measure the device performance, acts as a reference for other diagnostics [8] and can be used for machine protection, e.g. as a feedback for the neutral beam injectors. Classically there has been little interest in high bandwidth acquisition systems for the IED. However, in recent years research into Alfvèn Eigenmodes has benefited strongly from fast data acquisitions to resolve high frequency modes, e.g. Toroidal Alfvèn Eigenmodes (TAEs)[11].

On MAST-U the Thomson Scattering diagnostic will be the main benefactor of a high bandwidth IED system, since it uses the electron density for calibration[9]. It has been pointed out that the limited temporal resolution of the current interferometer system -generally $50 \mathrm{kHz}$ bandwidthleads to significant errors as interpolation is required[8].

The new MAST-U IED interferometer is based on field programmable gate arrays. FPGAs are increasingly being used for high-speed digital signal processing(DSP) purposes due to their high flexibility, which also enables reprogramming of firmware during experimental operations. The interferometer optical front-end is a $\mathrm{CO}_{2}-\mathrm{HeNe}$ two color infra-red interferometer operating at $10.6 \mu \mathrm{m}$ and $633 \mathrm{~nm}$ (see fig. 1). For a description of this part of the system the reader is kindly referred to a recent publication by O'Gorman et.al[5]. This paper presents the digitization back-end of the system.

The first section describes the hardware and setup of the system. This is followed by a more thorough description of the DSP firmware. Finally the diagnostic performance is presented.

\section{Diagnostic Hardware Setup}

\subsection{Hardware setup}

Figure 1 depicts the FPGA in relation to the entire diagnostic. It's main function is the digitization of the interferometer signals. The experimental data consists of 5 signals:

- the optical heterodyne detection signals from both the $\mathrm{CO}_{2}$ and the $\mathrm{HeNe}$ scene arm 
- the 2.36MHz rectangular reference signal from Agilent 5517B Interferometer

- the $40 \mathrm{MHz}$ sinusoidal reference signal from the $\mathrm{CO}_{2}$ acousto-optical modulator

- a trigger signal from the MAST-U experimental reference

Communication happens via a fiber optical duplex-link using the open Xilinx Aurora protocol. The link operates at $2 \mathrm{~Gb} / \mathrm{s}$ in duplex mode transferring 32bit packages carrying 16bits of data. The data is streamed with low latency to a FPGA based Aurora to PCIe bridge for storage and processing on a Linux PC or rerouting to the real-time-protection system. The aurora link is also used to receive control signals and status requests.

\subsection{Firmware}

The base board for the FPGA is a Simple PCIe Carrier (SPEC) board carrying a Xilinx Spartan-6 XC6SLX45T-3FGG484C FPGA. A 4 channel 100MSample/s(MS/s) 14bit analog-todigital converter (ADC) is connected to the SPEC via a FMC LPC connector. Both components are designed by the CERN Open Hardware Repository (OHWR)[7, 6]. Firmware design has been done using Xilinx ISE v14.7 and System Generator for MATLAB Simulink.

Figure 2 depicts the top level design of the firmware. The deserialization core has been taken and modified from an early version of the SPEC reference design.

The heart of the design is the processing core as indicated in fig. 2. The core evaluates the digitized signals for both lasers ensuring equal delay. Since the modulation frequencies of the $\mathrm{CO}_{2}$ interferometer is $40 \mathrm{MHz}$ the design directly heterodyne down converts the $\mathrm{CO}_{2}$ signal using a synthetic $36 \mathrm{MHz}$ sine and cosine generated on the FPGA. The down converted $4 \mathrm{MHz}$ intermediate frequency (IF) signal is then 5 -fold decimated using a $10 \mathrm{MHz}$ low-pass decimation filter implemented as an Equiripple finite impulse response (FIR) filter. This results in the inphase(I) and quadrature(Q) component of the respective signal.

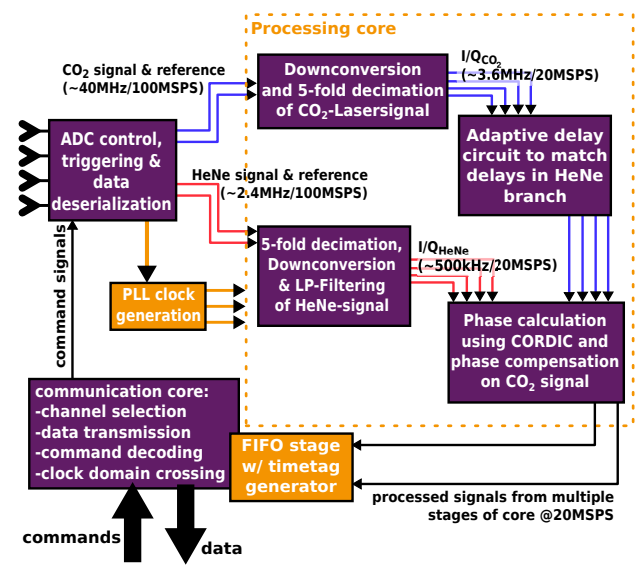

Figure 2: Top level of the diagnostic firmware. The top level design with the communication and the deserialization core is written in VHDL. The processing core is designed using System Generator for MATLAB Simulink. 
Since the HeNe signals are modulated at $2.36 \mathrm{MHz}$ the signal is first multiplexed and passed into a 5-fold decimator identical to the one in the $\mathrm{CO}_{2}$ arm. Past this point the processing is similar to the $\mathrm{CO}_{2}$ stage, albeit the signal is mixed with $1.8 \mathrm{MHz}$ signals resulting in a $600 \mathrm{kHz}$ IF.

To compensate for the difference in delays between the $\mathrm{CO}_{2}$ and the HeNe circuit the signals are matched with a separate core to minimize errors from timing mismatches. Subsequently the signals are fed into the phase measurement and correction core. This uses CORDIC, a very resource efficient phase rotation algorithm[3], to calculate the phase of each signal. After this the difference between respective scene and reference arm signals are calculated in a two-step process. First the scene and reference arm's phase wrap are synchronized before taking the difference using the fact that the phase will progress in one direction around the unit circle. This improves the effectiveness of the subsequent standard unwrapping algorithm. The two step unwrap increases the robustness of the system towards $2 \pi$ phase wraps with the limit being set by the IF. Finally the core conducts a streaming phase correction on the $\mathrm{CO}_{2}$ arm according to the standard correction formula[5]. The wavelengths are loaded from a register during the shot and can be adjusted to account for drifting wavelengths.

The final stage of the design is a communication core. Its purpose is to respond to request and control signals received via the fiber optic link, which it integrates using the Aurora $8 \mathrm{~b} / 10 \mathrm{~b}$ core provided by Xilinx[1]. In addition the core selects the channels that have been requested and formats them for sending.

\subsection{Aurora to PCIe bridge}

One of the reasons for not having the FPGA operate remotely is the sensitivity of the interferometer towards thermal fluctuations in the air. The remote setup required the design of an Aurora to PCIe bridge on the PC, which was implemented on a Xilinx SP605 FPGA[2]. It houses the same chip as the SPEC board and is equipped with a 1 lane PCIe Gen2 PHY.

The board has two functions:

1 : convert the aurora stream from the FPGA into a PC readable format. This is done using the commercial Xillybus core[4].

2 : fork the data stream and process it (decimation and evaluation) so it can be routed on to the real-time-protection system. This approach is preferable in terms of resource usage on the SPEC board although it introduces a few microseconds of latency.

\section{Diagnostic Performance}

Since at the time of writing the MAST Tokamak is in the process of being upgraded the diagnostic performance can only be measured using artificial data. For this an Agilent 33600A Series Waveform Generator was used to generate data simulating the diagnostic. In all cases the signals were chosen to be $900 \mathrm{mV}$ sinusoidals of the respective lasers IF frequency, albeit it should be noted that the HeNe reference input was a square waveform put through a $2.5 \mathrm{MHz}$ analog low pass filter. This reproduces the Agilent 5517B interferometer output signals. To improve performance the FPGA can only accept sinusoidal reference signals, hence the filter has the task of 


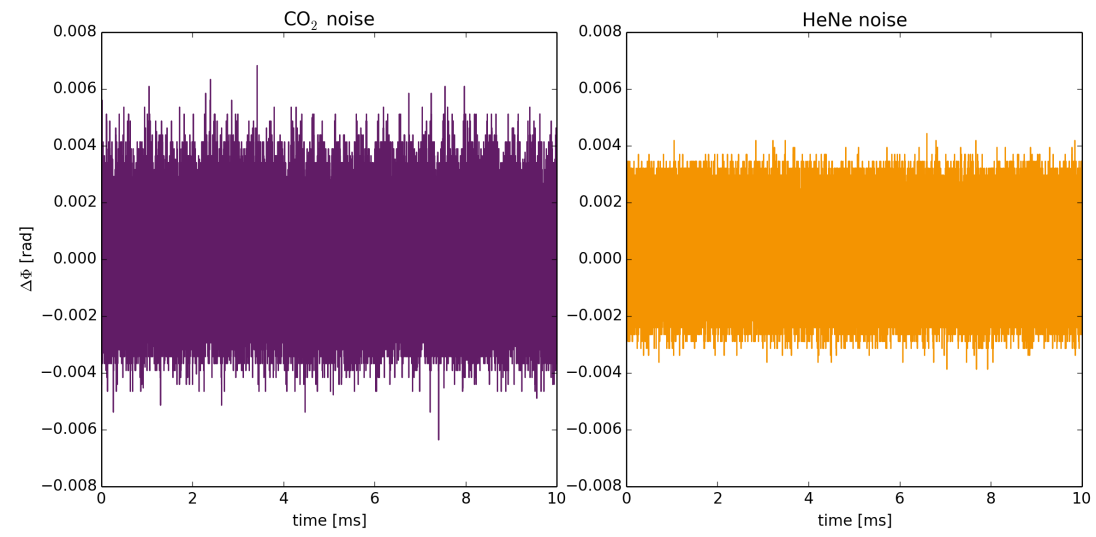

Figure 3: Raw noise measurement for both laser channels separately at full 20MS/s sampling rate.

returning the signal back to a sine at the cost of some extra noise. The total measurement time in all cases was $10 \mathrm{~ms}$.

\subsection{Phase noise}

The electrical or bit noise of the FPGA system was measured by supplying the system with the unmodified signals as described above, i.e. signal and reference input frequency of each respective input pair were identical.

Figure 3 shows the raw data of the $\mathrm{CO}_{2}$ and the HeNe arm measured in the above setup. Assuming all errors to be normally distributed one can find the error for the integral electron density by quadratic error propagation[10].

The error of the wavelengths are given by the lasers[5], which via error propagation results in the error for the wavelength ratio of under $3 \cdot 10^{-6}$ dominated by the error of the $\mathrm{CO}_{2}$ laser, however the main contribution to the density error is the phase error measured in fig. 3. In conclusion the density error due to the digitization system is found to be better than $\sigma_{n}=1.4 \cdot 10^{17} \mathrm{~m}^{-2}$ at full acquisition bandwidth.

\subsection{Bandwidth capabilities}

The design was evaluated for the possibility to measure high frequency perturbations of the input signals. This was done by phase modulating each signal input with a sinusoidal of a given frequency, whilst keeping the reference frequency fixed. The amplitude of the modulations was chosen to be $\pi / 3$ to prevent any additional effects from $2 \pi$ wraps.

Figure 4 depicts the phase modulated input signal over the respective Fourier peak amplitude. As can be seen the phase modulation can be detected up to a frequency of $4 \mathrm{MHz}$-the $\mathrm{CO}_{2} \mathrm{IF}$ frequency- after which the noise in the FT increases due to aliasing and the measurable peak amplitude decreases (with aliasing noise increasing) until it is lost in the aliasing noise at $6 \mathrm{MHz}$. The digitizer is thus capable of detecting multi-MHz modulations in the electron density. The roughness of the response curve can be attributed to unclean measurement environment and resonant effects occurring, however the level of sensitivity has been confirmed down to $100 \mathrm{~Hz}$ modulations. 


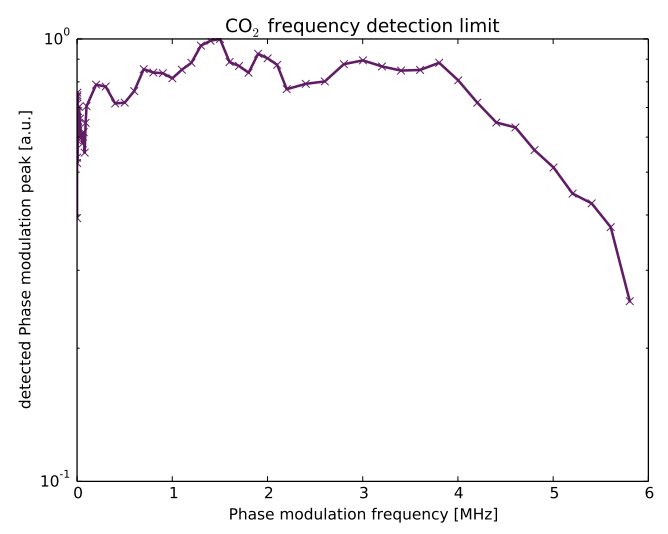

Figure 4: Bandwidth detection limit of the $\mathrm{CO}_{2}$ phase measurement arm. The input signal has been phase modulated and the respective peak in the Fourier spectrum been measured.

\subsection{Phase wrap stability}

$2 \pi$ phase wrap stability is a major concern for many two-color interferometer setups and an important quantity to be aware of in a real-time-protection system. The stability towards wraps was evaluated by changing the signal frequency to a fixed frequency different from the reference, thus simulating a constantly in-/decreasing plasma density or a constantly moving mirror. The measurements were then scanned for the lowest frequency at which wraps were missed. the $\mathrm{CO}_{2}$ and the HeNe arm had to be evaluated separately due to the differing IF and laser wavelength.

The measurement showed that the $\mathrm{CO}_{2}$ system will start missing phase wraps when signal and reference frequency differ by more than $4 \mathrm{MHz}$. This corresponds to a plasma density rate of change of almost $10^{21} \mathrm{~m}^{-2} / \mu \mathrm{s}$. These rates are an order of magnitude higher than the fastest events that can be expected in current and future Tokamaks, i.e. losing the entire core plasma density of a high density ITER shot $\left(\approx 10^{21}\right)$ within a microsecond, which is the speed of an ELM burst on MAST[8]. This would amount to a rate of density change of $10^{20} \mathrm{~m}^{-2} / \mu \mathrm{s}$, which is an order of magnitude faster than the rates expected during a disruption on JET[12]. The stability as a function of rate of density change is mainly significant for the $\mathrm{CO}_{2}$ line as it is more affected by the plasma dispersion. The HeNe limit for density changes are far beyond the $\mathrm{CO}_{2}$ ones.

The same data was evaluated in terms of rates of mirror speed. In this case the $\mathrm{HeNe}$ arm is the most affected as it uses a smaller wavelength. Due to the much lower IF the first phase wrap misses occur already at a total summed mirror speed only a few $100 \mathrm{~mm} / \mathrm{s}$. This would correspond to a $100 \mathrm{~Hz}$ sound wave of $1 \mathrm{~mm}$ amplitude. The most prominent candidate for vibrations is RR3 retro reflector, which for this reason has been isolated against mechanical vibrations as much as possible(see fig. 1)[5]. It is hard to estimate the vibration spectrum that will occur on the machine during operation in advance, hence further tests will have to be done once MAST-U is in the conditioning phase with the interferometer installed.

\subsection{Latency results}

As the design is meant to be integrated into the MAST-U real time protection system the latency of the design was measured. This was done by adding a Xilinx chipscope core to the 
Aurora-PCIe FPGA design to measure the time the interferometer FPGA would respond to a command sent via Aurora. The system latency was then calculated from the response times. First an ARM command was sent, which was acknowledged within $1 \mu \mathrm{s}$. Secondly a TRIGGER command was sent, which is not acknowledged by the FPGA, however it sets the next -and all subsequentdigitized samples valid enabling their processing and flagging them to be streamed. The TRIGGER package will pass through the same logic as the previous ARM package before an acknowledge, hence the latency of the system including the Aurora streaming logic is the time from half of the ARM acknowledge time to the first received data sample totalling less than $4 \mu \mathrm{s}$. This is an order of magnitude faster than the $30 \mu$ s minimum response time of the MAST-U RTP system.

\section{Summary \& Outlook}

An open hardware FPGA based digitization back-end for the new MAST-U two-color integral electron density interferometer has been designed. The design is capable of detecting plasma density perturbations beyond $4 \mathrm{MHz}$ with an accuracy of $0.002 \mathrm{rad}$ phase-shift corresponding to $10^{17} \mathrm{~m}^{-2}$ at full 20MS/s digitization bandwidth for an arbitrary length shot. This is an improvement over the number cited in the initial presentation of the optical setup[5]. The system latency of $3 \mu$ s enables the integration of the system into a machine real-time-protection system, albeit with the full acquisition bandwidth the amount of data generated is more than can be handled by a RTP system and should thus be decimated accordingly. Phase stability has also been shown to be an order of magnitude higher than requirements found in current and future Tokamak devices.

Testing of the system however could so far only been done with artificial data due to the MAST-U upgrade being in progress. The current plan is to implement the digitization system parasitically on a different machine operating with a similar system. Due to different IF frequencies it is possible that the design requires changing from an IQ demodulation via downmixing approach to a Hilbert transformation to generate the Q signal. The rest of the system would be untouched. This approach would also be possible to implement for the MAST system, however due to the higher filter complexity has not been chosen as an initial option. In terms of errors it is expected that such a change would at best improve the error and bandwidth capabilities of the system. It would also improve the $2 \pi$ phase wrap stability against mechanical vibrations by a factor of 4 .

\section{Acknowledgements}

This work is funded by Durham University and EPSRC grant EP/L01663X/1.

\section{References}

[1] Xilinx Inc. Aurora 8b/10b. web resource, 122014.

[2] Xilinx Inc. Spartan-6 fpga sp605 evaluation kit. web resource, 122014.

[3] B. Lakshmi and A. S. Dhar. Cordic architectures: A survey, 2010.

[4] Xillybus Ltd. An fpga ip core for easy dma over pcie with windows and linux. web resource, 122015. 
[5] T. O’Gorman, G. Naylor, R. Scannell, G. Cunningham, K. J. Brunner, R. Martin, and D. Croft. Design of a real-time two-color interferometer for mast upgrade. Review of Scientific Instruments, 85(11):-, 2014.

[6] Open Hardware Repository. Fmc adc 100m 14b 4cha. web resource, 82014.

[7] Open Hardware Repository. Simple pcie carrier v4 (spec). web resource, 82014.

[8] R Scannell, A Kirk, N Ben Ayed, P G Carolan, G Cunningham, J McCone, S L Prunty, and M J Walsh. Experimental investigation into elm filament formation on mast. Plasma Physics and Controlled Fusion, 49(9):1431, 2007.

[9] R. Scannell, M. J. Walsh, P. G. Carolan, N. J. Conway, A. C. Darke, M. R. Dunstan, D. Hare, and S. L. Prunty. Enhanced edge thomson scattering on mast. Review of Scientific Instruments, 77(10):-, 2006.

[10] M. A. Van Zeeland, R. L. Boivin, D. L. Brower, T. N. Carlstrom, J. A. Chavez, W. X. Ding, R. Feder, D. Johnson, L. Lin, R. C. O’Neill, and C. Watts. Conceptual design of the tangentially viewing combined interferometer-polarimeter for iter density measurements. Review of Scientific Instruments, 84(4):-, 2013.

[11] M. A. Van Zeeland and G. J. Kramer. Measurements of alfven eigenmodes in fusion plasmas. AIP Conference Proceedings, 988(1):103-109, 2008.

[12] J.A. Wesson, R.D. Gill, M. Hugon, F.C. Schüller, J.A. Snipes, D.J. Ward, D.V. Bartlett, D.J. Campbell, P.A. Duperrex, A.W. Edwards, R.S. Granetz, N.A.O. Gottardi, T.C. Hender, E. Lazzaro, P.J. Lomas, N. Lopes Cardozo, K.F. Mast, M.F.F. Nave, N.A. Salmon, P. Smeulders, P.R. Thomas, B.J.D. Tubbing, M.F. Turner, and A. Weller. Disruptions in jet. Nuclear Fusion, 29(4):641, 1989. 